Lattice super-Yang-Mills

\title{
using domain wall fermions in the chiral limit
}

\author{
Joel Giedt* \\ Department of Physics, Applied Physics and Astronomy, \\ Rensselaer Polytechnic Institute, 110 8th Street, Troy NY 12065 USA \\ Richard Brower $\dagger$ \\ Physics Department, Boston University, \\ 590 Commonwealth Avenue, Boston MA 02215 \\ Simon Catteral $\ddagger$ \\ Department of Physics, Syracuse University, Syracuse, NY 13244 USA \\ George T. Fleming $\S$ \\ Department of Physics, Sloane Laboratory, \\ Yale University, New Haven, Connecticut 06520, USA \\ Pavlos Vranas \\ Physical Sciences Directorate, Lawrence Livermore National Laboratory, \\ 7000 East Ave., Livermore, CA 94550
}

(Dated: Dec. 2, 2008) 


\begin{abstract}
Lattice $\mathcal{N}=1$ super-Yang-Mills theory formulated using Ginsparg-Wilson fermions provides a rigorous non-perturbative definition of the continuum theory that requires no fine-tuning as the lattice spacing is reduced to zero. Domain wall fermions are one explicit scheme for achieving this and using them we have performed large scale Monte Carlo simulations of the theory for gauge group $S U(2)$. We have measured the gaugino condensate, static potential, Creutz ratios and residual mass for several values of the domain wall separation $L_{s}$, four-dimensional lattice volume, and two values of the bare gauge coupling. With this data we are able to extrapolate the gaugino condensate to the chiral limit, to express it in physical units, and to establish important benchmarks for future studies of super-Yang-Mills on the lattice.
\end{abstract}

PACS numbers: 11.15.Ha,11.30.Pb

*Electronic address: giedtj@rpi.edu

$\dagger$ Electronic address: brower@bu.edu

${ }^{\ddagger}$ Electronic address: smc@physics.syr.edu

$\S$ Electronic address: George.Fleming@yale.edu

『Electronic address: vranasp@llnl.gov 


\section{INTRODUCTION}

The lattice formulation of supersymmetric theories necessarily breaks most or all ${ }^{1}$ of the continuum supersymmetry, since the translation group, a subgroup of the super-Poincaré group, is broken to a discrete subgroup. This is intimately related to the failure of the Leibniz rule on the lattice [3]. Hence all relevant and marginal operators that are allowed by lattice symmetries will be generated radiatively, so that generically the long distance effective theory will have supersymmetry badly broken.

On the other hand, the only relevant or marginal operator allowed in a gauge invariant lattice formulation of pure $\mathcal{N}=1$ super-Yang-Mills [4] (SYM) with hypercubic symmetry is the gaugino mass term, as was emphasized long ago in the analysis of [5]. By using Ginsparg-Wilson fermions [6], the associated lattice chiral symmetry [7] protects against additive renormalizations of the gaugino mass in the continuum limit. Hence the desired continuum theory is obtained without fine-tuning of counterterms, merely by setting the bare fermion mass to zero.

A viable lattice formulation provides nonperturbative information regarding SYM, the foundation for all nonabelian four-dimensional supersymmetric gauge theories. Given the unique features of supersymmetric theories, it makes sense to study their strong dynamics by as many means as possible. It is quite exciting that the lattice approach can be brought to bear on $\mathcal{N}=1 \mathrm{SYM}$ in a rigorous and reliable way, since it would be enlightening to study the nonperturbative aspects of this theory in detail using numerical techniques. For instance, information on the spectrum and renormalization of nonholomorphic operators would be welcome.

It is essential to quantify the size of lattice artifacts, since these lead to deviations from the continuum physics. One of the purposes of the present study is to determine the regime of lattice parameters where continuum SYM makes its appearance (to a good approximation), and to characterize the difficulty of performing Monte Carlo studies in that limit, given the algorithms and computing resources that are currently available. The study that we present is representative of what can be achieved with a dedicated world-class supercom-

\footnotetext{
${ }^{1}$ In cases with extended supersymmetry it is sometimes possible to preserve a nilpotent subalgebra (see for example the reviews [1, 2] and references therein). For minimal supersymmetry, such as is studied here, all of the generators are broken.
} 
puting resource - of order 21 Teraflop/s × year (30 Million IBM BlueGene/L core hours) on Rensselaer's Computational Center for Nanotechnology Innovation - and highly optimized parallel code (a modified version of the Columbia Physics System). It will be seen that we are able to obtain reliable extrapolations to the chiral limit (vanishing gaugino mass), but that the study was quite demanding and could not have been performed with anything less than the resources just described.

Whereas much is known about the vacuum structure of SYM by continuum methods [8, 9, 10, 11, 12, 13], nothing is known about its long distance dynamics and spectrum. Furthermore, lattice methods have the ability to reveal far more detail about the vacuum, as one can subject it to a configuration by configuration analysis, as has been done for pure Yang-Mills with considerable success. Finally, we are interested in the effects of a small, nonzero gaugino mass in the nonperturbative regime. The explicit, but controllable, chiral symmetry breaking of the fermion discretization we use here allows us to explore the impact of a small gaugino "soft mass" on nonperturbative quantities such as the condensate, the string tension and the shape of the static potential. This is in the spirit of a large number of proposals made over the last several years [14, 15, 16, 17, 18, 19, 20]. Ultimately, the impact of nonzero gaugino mass on the spectrum of bound states will emerge from high statistics studies that are beyond the scope of the present work. It will then be possible to compare the lattice data to the references just cited.

One implementation of Ginsparg-Wilson fermions is domain wall fermions (DWF) [21, 22] in the limit of infinite separation between the walls, $L_{s} \rightarrow \infty$. Besides the absence of nonperturbative fine-tuning of the gaugino mass, DWF have the advantage that the fermion measure is real, positive and the square root of the determinant which enforces the Majorana condition is analytic with a phase that is independent of the gauge fields [23, 24]. These three features are all lacking in the Wilson fermion formulation that was applied in the only concerted effort to date to study SYM on the lattice by the DESYMünster-Roma collaboration [25, 26, 27, 28, 29, 30] and to a lesser extent Donini et al. [31]. (Recently, this program has been revived [32].) Our research, which has already appeared in preliminary form [33], is in some sense a continuation of the work of Fleming, Kogut and

\footnotetext{
${ }^{2}$ This is the actual computing power brought to bear. The theoretical computing power utilized was ten times this.
} 
Vranas (FKV) [34] who pioneered the use of DWF for studying $\mathcal{N}=1$ SYM. Similar work has been initiated by Endres [35]. What sets the present study apart is that an extensive scan of the domain wall separation $L_{s}$ and measurement of the residual chiral symmetry breaking mass $m_{\text {res }}$ was done at two values of the bare lattice gauge coupling $\left(\beta=4 / g^{2}=2.3\right.$ and 2.4) and spatial/temporal volumes $\left(L^{3}=8^{3}\right.$ and $\left.16^{3} ; T=16,32\right)$. This has allowed us to obtain a reliable chiral extrapolation $\left(m_{\mathrm{res}} \rightarrow 0\right)$, and a preliminary view on what occurs as we take the continuum, theormodynamic limit $(\beta, L, T \rightarrow \infty)$.

The lattice formulation that is used in this study has already been described by FKV [34]; it is reviewed in Appendix A. In brief, the lattice employs Shamir DWF [22] in the adjoint representation of $\mathrm{SU}(2)$, and the one-plaquette Wilson gauge action. The Majorana condition is imposed through a square root on the fermion determinant, which as mentioned above is analytic and introduces no gauge field dependent sign ambiguity [23, 24].

All results reported in this article utilize a domain wall height $m_{0}=1.9$, as in the FKV simulations, though we will comment briefly on some tests we did at other values of $m_{0}$. Lattice configurations were generated with a dynamical fermion mass $m_{f}=0$, so that the finite size of the fifth dimension, parameterized by $L_{s}$, was the sole infrared regulator, through the corresponding additive mass correction $m_{\text {res }}$ (reviewed in Appendix B), which is a measure of residual chiral symmetry breaking [36]. One does not expect an optimal value of $m_{0}$ to exist [37], but for stronger couplings, the range is rather narrow. Our $m_{\text {res }}$ measurements below show that we are in the correct phase - the explicit chiral symmetry breaking decreases as $L_{s}$ is increased - for $m_{0}=1.9$. Finally, using the lattice configurations that we generated, we computed $m_{\text {res }}$ for DWF propagators with other values of $m_{0}$. We found that $m_{\text {res }}$ could be lowered slightly by increasing $m_{0}$ toward the critical value 2 , and that decreasing $m_{0}$ from 1.9 increased $m_{\text {res }}$. The decrease in $m_{\text {res }}$ by increasing $m_{0}$ was not significant, so we did not pursue the issue further.

In Section [II we give our results for the bare gaugino condensate for various couplings $\beta=4 / g^{2}$ and domain wall separations $L_{s}$. Then in Section III we summarize our findings for "gluonic" observables (i.e., those related to the nonabelian gauge bosons), principally the string tension and thereby the Sommer parameter $r_{0} / a$. Next in Section [V] we discuss the chiral extrapolations of the gaugino condensate based on our simulation results. We conclude in Section V. In addition to the two appendices mentioned above, Appendix C describes technical aspects of the simulation that may be of interest. 


\begin{tabular}{|c|c|c|c|c|c|}
\hline$\beta$ & 2.1 & 2.2 & 2.3 & 2.4 & 2.5 \\
\hline$\langle\bar{\lambda} \lambda\rangle a^{3}$ & $0.007494(5)$ & $0.007719(5)$ & $0.007051(5)$ & $0.00499(6)$ & $0.003043(5)$ \\
\hline
\end{tabular}

TABLE I: A scan of the condensate versus $\beta$ for the $16^{3} \times 32$ lattice with domain wall separation $L_{s}=16$.

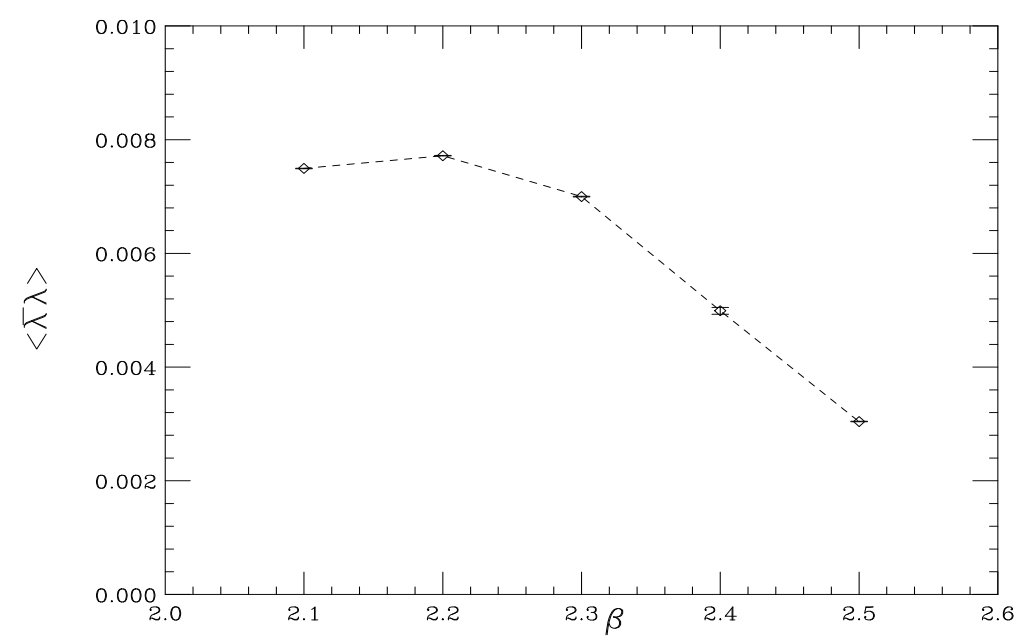

FIG. 1: Condensate vs. $\beta$ for $16^{3} \times 32$ lattice with $L_{s}=16$.

\section{BARE GAUGINO CONDENSATE}

We have validated our simulation code by comparing to FKV at several points. We obtained results that agree with FKV, to within 1\% statistical errors.

A summary of all results obtained here for the gaugino condensate $\langle\bar{\lambda} \lambda\rangle$ is given in Tables [, II and III. The residual chiral symmetry breaking is parameterized through $m_{\text {res }}$ [36], which we briefly review in Appendix B. Measurements were conducted on large and small lattice volumes; it can be seen that in lattice units the finite-size dependence is mild or insignificant for $\beta=2.3$ but quite noticeable for $\beta=2.4$. This is sensible, given that $\beta=2.4$ corresponds to a finer lattice spacing, and hence the physical volumes are smaller. Simulations on $16^{3} \times 32$ volumes with $L_{s}=48$ are in progress and will be presented elsewhere.

For the $L_{s}=16$ lattices, which are relatively inexpensive, a scan over $\beta$ was performed, with results given in Table I and shown in Fig. 1. The vanishing extrapolated value at $\beta \sim 2.7$ is apparently due to finite-size effects that cause the system to deconfine.

We have measured the condensate at other values of $L_{s}$ using a sea- $L_{s} /$ valence- $L_{s}$ ap- 


\begin{tabular}{|c|c|c|c|c|c|}
\hline$V \times T$ & $L_{s}$ & $m_{\mathrm{res}} a$ & $\langle\bar{\lambda} \lambda\rangle a^{3}$ & $m_{\mathrm{res}} r_{0}$ & $\langle\bar{\lambda} \lambda\rangle r_{0}^{3}$ \\
\hline $8^{3} \times 8$ & 16 & $0.158(5)$ & $0.00711(7)$ & - & - \\
$8^{3} \times 32$ & 16 & $0.181(3)$ & $0.00703(4)$ & $0.75(13)$ & $0.51(27)$ \\
$16^{3} \times 32$ & 16 & $0.184(2)$ & $0.007051(5)$ & $0.668(10)$ & $0.337(11)$ \\
\hline $8^{3} \times 32$ & 24 & $0.1541(15)$ & $0.005112(8)$ & $0.610(97)$ & $0.32(15)$ \\
$16^{3} \times 32$ & 24 & $0.1564(17)$ & $0.005321(9)$ & $0.546(55)$ & $0.226(68)$ \\
\hline $8^{3} \times 32$ & 32 & $0.1319(12)$ & $0.004321(11)$ & $0.501(69)$ & $0.24(10)$ \\
$16^{3} \times 32$ & 32 & $0.143(2)$ & $0.00445(2)$ & $0.483(58)$ & $0.172(61)$ \\
\hline $8^{3} \times 32$ & $40(\mathrm{I})$ & $0.1183(54)$ & $0.00383(3)$ & - & - \\
\hline $8^{3} \times 16$ & 48 & $0.1043(17)$ & $0.003563(20)$ & $0.361(31)$ & $0.148(37)$ \\
$8^{3} \times 32$ & 48 & $0.1071(10)$ & $0.003551(11)$ & $0.409(31)$ & $0.198(45)$ \\
\hline $8^{3} \times 32$ & 64 & $0.08864(84)$ & $0.003164(10)$ & $0.300(35)$ & $0.122(42)$ \\
\hline
\end{tabular}

TABLE II: The gaugino condensate $\langle\bar{\lambda} \lambda\rangle$ and residual mass $m_{\text {res }}$ for various lattice sizes and $L_{s}$ values, all at $\beta=2.3$. The $L_{s}=40$ value, with an "(I)" after it, is obtained by the interpolation method described in the text, and summarized in Table IV Values in units of the Sommer parameter $r_{0}$ are also shown, for those cases where the potential was measured (in particular, for all points that are included in the chiral extrapolation fit). The $L_{s}=16$ data was not included in the linear chiral extrapolation fit, because these points had too much curvature (with respect to $\left.m_{\text {res }} a\right)$ associated with them.

proach. The condensate was measured using DWF with $L_{s}^{\mathrm{val} .}$ on top of dynamical lattices produced using a nearby $L_{s}^{\text {sea }}$. Performing this for $L_{s}^{\text {sea }}$ values on either side of $L_{s}^{\text {val. yields }}$ robust interpolated (I) results, as can be seen in Table IV. These are then used in our fits of $\langle\bar{\lambda} \lambda\rangle$ vs. $m_{\text {res }}$, together with the strictly dynamical $\left(L_{s}^{\text {val. }}=L_{s}^{\text {sea }}\right)$ measurements of Tables 【I and III. Interpolations ("I") are taken halfway between the results. Half the difference plus the statistical errors added in quadrature is used as an error estimate for the interpolation.

We also use the results of Section IIIB below to express $m_{\text {res }}$ and $\langle\bar{\lambda} \lambda\rangle$ in terms of the Sommer scale $r_{0}$ [38]. Note that the $\beta=2.4$ value of $m_{\text {res }} r_{0}$ at $L_{s}=48$ indicates that the effective gaugino mass (which should be approximately equal to $m_{\text {res }}$ ) is roughly 1/4 the inverse Sommer scale, so that we are beginning to enter the chiral regime where 


\begin{tabular}{|c|c|c|c|c|c|}
\hline$V \times T$ & $L_{s}$ & $m_{\mathrm{res}} a$ & $\langle\bar{\lambda} \lambda\rangle a^{3}$ & $m_{\mathrm{res}} r_{0}$ & $\langle\bar{\lambda} \lambda\rangle r_{0}^{3}$ \\
\hline $8^{3} \times 32$ & 16 & $0.080(2)$ & $0.004839(15)$ & $0.547(30)$ & $1.55(22)$ \\
$16^{3} \times 32$ & 16 & $0.0969(8)$ & $0.00499(6)$ & $0.5355(66)$ & $0.842(25)$ \\
\hline $8^{3} \times 32$ & 24 & $0.0601(15)$ & $0.003293(17)$ & $0.417(26)$ & $1.10(18)$ \\
$16^{3} \times 32$ & 24 & $0.0838(17)$ & $0.00389(8)$ & $0.385(35)$ & $0.38(10)$ \\
\hline $16^{3} \times 32$ & $28(\mathrm{I})$ & $0.0721(33)$ & $0.003452(45)$ & - & - \\
\hline $8^{3} \times 32$ & 32 & $0.0486(12)$ & $0.00269(2)$ & $0.296(15)$ & $0.61(08)$ \\
$16^{3} \times 32$ & 32 & $0.0653(15)$ & $0.003330(12)$ & $0.313(33)$ & $0.37(11)$ \\
\hline $8^{3} \times 32$ & $40(\mathrm{I})$ & $0.0390(24)$ & $0.00234(8)$ & - & - \\
\hline $8^{3} \times 32$ & 48 & $0.0328(9)$ & $0.002165(18)$ & $0.224(17)$ & $0.69(15)$ \\
\hline
\end{tabular}

TABLE III: Results similar to Table II, except that these are for $\beta=2.4$.

\begin{tabular}{|c|c|c|c|c|c|c|}
\hline$\beta$ & $V$ & $T$ & $L_{s}^{\text {val. }}$ & $L_{s}^{\text {sea. }}$ & $m_{\mathrm{res}} a$ & $\langle\bar{\lambda} \lambda\rangle a^{3}$ \\
\hline 2.3 & $8^{3}$ & 32 & 40 & 32 & $0.117(4)$ & $0.003818(9)$ \\
2.3 & $8^{3}$ & 32 & 40 & 48 & $0.1196(10)$ & $0.003843(9)$ \\
2.3 & $8^{3}$ & 32 & 40 & $\mathrm{I}$ & $0.1183(54)$ & $0.00383(3)$ \\
\hline 2.4 & $16^{3}$ & 32 & 28 & 24 & $0.0707(13)$ & $0.003407(3)$ \\
2.4 & $16^{3}$ & 32 & 28 & 32 & $0.0734(15)$ & $0.003496(3)$ \\
2.4 & $16^{3}$ & 32 & 28 & $\mathrm{I}$ & $0.0721(33)$ & $0.003452(45)$ \\
\hline 2.4 & $8^{3}$ & 32 & 40 & 32 & $0.0381(10)$ & $0.002284(13)$ \\
2.4 & $8^{3}$ & 32 & 40 & 48 & $0.0398(11)$ & $0.002397(17)$ \\
2.4 & $8^{3}$ & 32 & 40 & $\mathrm{I}$ & $0.0390(24)$ & $0.00234(8)$ \\
\hline
\end{tabular}

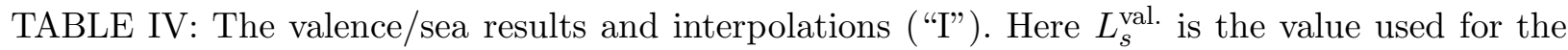
measurements and $L_{s}^{\text {sea }}$ is the value used in the dynamical fermion simulations.

supersymmetry is well approximated. On the other hand, it can be seen that $m_{\text {res }} r_{0}$ is unpleasantly large for $\beta=2.3$ with $L_{s} \leq 32$, and likewise the condensate in physical units is small compared to the $\beta=2.4$ results. Clearly $\beta=2.3$ is further away from the supersymmetric limit due to the coarser lattice. On the other hand it can be seen that the $\beta=2.4$ data shows a marked volume dependence due to the smaller physical "box" that 
the states must squeeze into.

We note that in the present context the $m_{\text {res }}$ measurement coming from the midpoint "pion" propagator calculation is a quenched probe of explicit chiral symmetry breaking, since in the dynamical theory (i.e., the one pertaining to the lattice action that is used to generate configurations) we do not have two "valence quarks" and a nonanomalous continuous chiral symmetry that would give rise to pseudo-Nambu-Goldstone bosons. Rather, the (adjoint) pions that are measured in the midpoint calculation of $m_{\text {res }}$ are pseudo-Nambu-Goldstone bosons of an $S U(4 \mid 3)$ graded chiral symmetry that is spontaneously broken in the chiral limit, and explicitly broken at finite $L_{s}$, as we now explain.

The $S U(4)$ subalgebra of the graded Lie algebra $S U(4 \mid 3)$ is associated with the gaugino (a Majorana fermion) plus three quenched Majorana fermion probes. The total of four Majorana fermions with degenerate mass is equivalent to two Dirac fermions in the adjoint representation, with a resulting $S U(4)$ chiral flavor symmetry in the chiral limit. The fact that three of the four Majorana degrees of freedom are quenched is equivalent to introducing three Majorana ghosts, also with the same mass. As in partially quenched QCD, the ghosts cancel the contribution of the nondynamical fermions to the functional integration measure. Also analogous to quenched QCD, there is a graded Lie algebra that relates the fermion and ghost fields, namely $S U(4 \mid 3)$ in the present case. The PCAC mass $\left(m_{\text {res }}\right)$ associated with this $N_{f}=2$ adjoint-Dirac fermion chiral symmetry breaking is a good probe of the DWF chiral limit, for the same reasons that it is a solid tool in quenched DWF-QCD studies. Investigations of the effective theory description of the $S U(4 \mid 3)$ algebra, from the theoretical perspective as it relates to DWF, are in progress [39]. Finally we note that in recent spectrum studies of one-flavor QCD, a similar non-singlet flavor current was utilized with success [40].

\section{GLUONIC OBSERVABLES}

One of the interesting features of SYM is that it is a theory with dynamical fermions that do not screen static sources in the fundamental representation. In contrast to QCD, it has true confinement in the sense of an area law and no string breaking. (Recall that the gauge action is expressed in terms of fundamental links, so we are not studying the $S U(2) / Z_{2}=S O(3)$ gauge theory, wherein fundamental sources would have an ambiguous meaning.) On the lattice, we can therefore study a very interesting static potential-one 


\begin{tabular}{|l|l|l|l|l|l|l|}
\hline$\beta$ & $\chi(1,1)$ & $\chi(2,2)$ & $\chi(3,3)$ & $\chi(4,4)$ & $\chi(5,5)$ & $\chi(6,6)$ \\
\hline 2.1 & $0.6423(6)$ & $0.5242(16)$ & $0.459(7)$ & $0.4(2)$ & - & - \\
\hline 2.2 & $0.580(2)$ & $0.427(5)$ & $0.330(10)$ & $0.12(5)$ & - & - \\
\hline 2.3 & $0.51051(11)$ & $0.3091(4)$ & $0.1997(12)$ & $0.163(11)$ & $0.17(7)$ & - \\
\hline 2.4 & $0.45966(7)$ & $0.2346(3)$ & $0.1227(9)$ & $0.078(3)$ & $0.052(14)$ & $0.19(7)$ \\
\hline 2.5 & $0.42493(8)$ & $0.1969(3)$ & $0.0896(5)$ & $0.0507(17)$ & $0.036(7)$ & - \\
\hline
\end{tabular}

TABLE V: Creutz ratios for the $16^{3} \times 32 \times 16$ lattice.

with chiral fermions and a nonvanishing string tension. These features of SYM will be presented here, illustrating the special ability of the lattice approach: to conduct detailed studies of the nonperturbative aspects of the theory that the continuum methods cannot touch upon.

\section{A. Creutz ratios}

Here we look at Creutz ratios [41] as a probe of the string tension in lattice units, $\sigma a^{2}$, as well as to delineate the scaling regime where the continuum limit may be extracted. Results for the $16^{3} \times 32 \times 16$ lattice are shown in Table $\nabla$ and Fig. 2. Although the errors are somewhat large, scaling is clearly setting in at around $\beta \sim 2.4$ as can be seen by the $\chi(4,4)$ ratios, which lie quite close to the 2-loop curve. Much beyond that $\beta$, finite size effects will take over and it is necessary to move to a larger lattice. For this reason, most of our simulations have been performed at $\beta=2.3$ and $\beta=2.4$.

\section{B. Static potential}

The static potential was obtained by measuring Wilson loops with one side of length $t$ in the temporal direction, according to standard methods. Having obtained $V(r) a$ from fitting the exponential decay in time, we next analyze the potential in terms of the distance $r a$. We fit the data to the standard form

$$
V(r) a=V_{0} a+\sigma a^{2}(r / a)-\frac{\alpha}{r / a}
$$




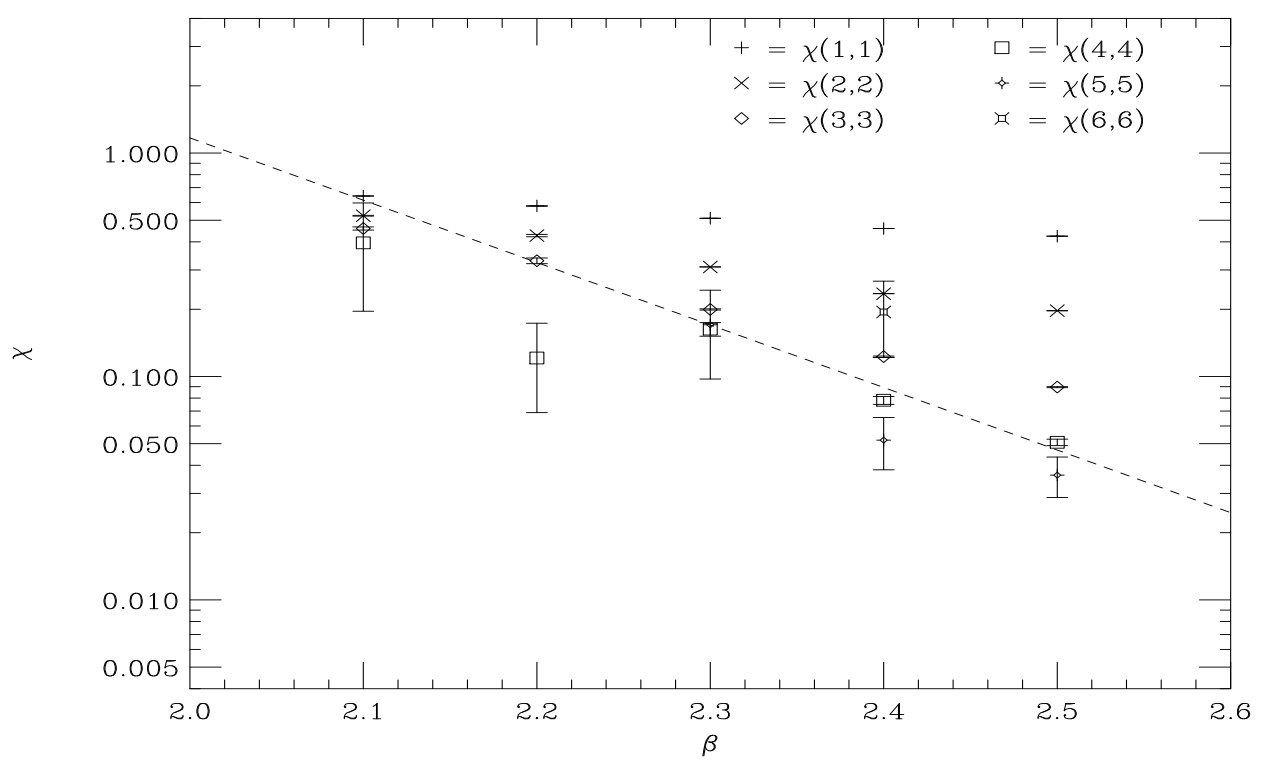

FIG. 2: Creutz ratios for the $16^{3} \times 32 \times 16$ lattice. The dashed line indicates the 2-loop prediction for the dependence $a^{2}(\beta)$, apart from an overall normalization that is determined by requiring that the curve pass through the data.

as was also done by the DESY-Münster-Roma collaboration in [25], and in the recent work [35]. We obtain the Sommer parameter $r_{0} / a$ from this fit, using the formula

$$
\frac{r_{0}}{a}=\sqrt{\frac{1.65-\alpha}{\sigma a^{2}}} .
$$

This approach to the determination of $r_{0} / a$ has some sensitivity to the range of radii that is fit, and obviously depends on what form we assume for $V(r) a$. The force method of Sommer [38] improves on this by avoiding a static potential fit, relying instead on treelevel improved finite differences. Preliminary studies that we have conducted indicate that smearing of gauge links and higher statistics will be indisposable to a successful application of the method, and so we will perform this in a future study.

The results of our static potential fit and the derived quantities are presented in Tables VI and VII. Note that the values of the string tension in physical units, $\sigma r_{0}^{2}$, are quite uniform for fixed $\beta$, and have small errors. For the $L=16$ results, the fits were also done using the same set of Wilson loops as in the $L=8$ case, denoted " $L=8$ method", so that dependence choice of Wilson loops could be controlled for, and therefore ruled out as a spurious source of finite size dependence. It also gives a clearer picture of the systematic uncertainties associated with the measurement and fitting methods for the static potential. 
These results are to be compared with Table 1 of [35]. There, a nonzero fermion mass $m_{f}=0.02$ was used, $L_{s}=16$, and somewhat different fit ranges for $r, t$ were employed. In particular, our fits include the points $r / a=1, \sqrt{2}$, which have very small errors and thus strongly influence the fit. Thus for $L_{s}=16$ we have also performed a fit with $r_{\min }=\sqrt{3}$ as was done in [35], as can be seen in the second $L_{s}=16$ entry for $\beta=2.3,2.4$ in Tables VI and VII. Our results for the fit quantities at $\beta=2.3$ are in good agreement once this restriction is imposed. For our other $L_{s}$ values we have far fewer samples, as larger $L_{s}$ comes at greater computing cost. The degradation of statistical errors that results if we exclude the $r=1, \sqrt{2}$ points is unacceptable, which is why we do not quote results with the same $r_{\min }=\sqrt{3}$ as [35] for the other $L_{s}$ values. On the other hand, it can be seen from the $L_{s}=16$ results that the choice of $r_{\min }$ only has a $10 \%$ effect on the $r_{0} / a$ estimate, so that the choice of $r_{\min }$ is not crucial to the broad picture that we are after in this preliminary work. The $\beta=2.4$ results are also in reasonable agreement with [35], comparing to the numbers we obtain at $16^{3} \times 32, L_{s}=16$, with $r_{\min }=\sqrt{3}$, and keeping in mind the nonzero $m_{f}$ in [35].

Above, we have used the results of Tables VI and VII to scale the residual mass and condensate to $r_{0}$ units. (Note that the $r_{0} / a$ values with identical lattice parameters were used in this procedure, rather than a uniform $r_{0} / a$ value across all $m_{\text {res }}$ and $\langle\bar{\lambda} \lambda\rangle$.) With the string tension in hand, we now see that the energy scale of confinement $\sqrt{\sigma r_{0}^{2}} \approx 1.4$ lies above the explicit chiral symmetry breaking scale $m_{\text {res }} r_{0}$ by a factor of 1.8 to 3.8 for $\beta=2.3$, and 3.0 to 5.2 for $\beta=2.4$. This is consistent with the observation that the string tension results in Tables VI and VII are insensitive to the range of $L_{s}$ values displayed there, when expressed in physical units $\left(\sigma r_{0}^{2}\right)$. That is, confinement dynamics are to a good approximation decoupled from the explicit chiral symmetry breaking. Since the lowest lying excitations of SYM are glueballs and superpartners, the gap associated with confinement should also decouple these states from the explicit chiral symmetry breaking. Thus it appears that we are well into the regime where the spectrum reflects supersymmetry, and it will be quite interesting to examine the spectrum in order to check whether or not this is true something we will do in future work. In addition, it gives us confidence that we are performing the chiral extrapolation of the condensate (next section) correctly, where the data points are dominated by the physics of the supersymmetric theory. 


\begin{tabular}{|c|c|c|c|c|c|c|c|}
\hline$V \times T$ & $L_{s}$ & $V_{0} a$ & $\sigma a^{2}$ & $\alpha$ & $r_{0} / a$ & $\sigma r_{0}^{2}$ & method \\
\hline $8^{3} \times 32$ & 16 & $0.717(83)$ & $0.074(28)$ & $0.368(55)$ & $4.16(73)$ & $1.282(55)$ & $L=8$ \\
$16^{3} \times 32$ & 16 & $0.6533(80)$ & $0.1004(25)$ & $0.3271(57)$ & $3.630(39)$ & $1.3229(57)$ & $L=16$ \\
$16^{3} \times 32$ & 16 & $0.489(45)$ & $0.1367(88)$ & $0.159(55)$ & $3.303(50)$ & $1.491(55)$ & $L=16, r \geq \sqrt{3}$ \\
\hline $8^{3} \times 32$ & 24 & $0.718(89)$ & $0.082(30)$ & $0.371(60)$ & $3.96(63)$ & $1.279(60)$ & $L=8$ \\
$16^{3} \times 32$ & 24 & $0.752(70)$ & $0.102(24)$ & $0.411(46)$ & $3.49(35)$ & $1.239(46)$ & $L=16$ \\
$16^{3} \times 32$ & 24 & $0.696(67)$ & $0.119(22)$ & $0.372(46)$ & $3.27(24)$ & $1.278(46)$ & $L=8$ \\
\hline $8^{3} \times 32$ & 32 & $0.748(82)$ & $0.087(27)$ & $0.400(55)$ & $3.80(52)$ & $1.250(55)$ & $L=8$ \\
$16^{3} \times 32$ & 32 & $0.745(90)$ & $0.109(31)$ & $0.412(59)$ & $3.38(40)$ & $1.238(59)$ & $L=16$ \\
$16^{3} \times 32$ & 32 & $0.635(50)$ & $0.146(17)$ & $0.338(33)$ & $3.00(14)$ & $1.312(33)$ & $L=8$ \\
\hline $8^{3} \times 16$ & 48 & $0.706(68)$ & $0.107(22)$ & $0.372(47)$ & $3.46(29)$ & $1.278(47)$ & $L=8$ \\
$8^{3} \times 32$ & 48 & $0.768(47)$ & $0.085(15)$ & $0.414(33)$ & $3.82(29)$ & $1.236(33)$ & $L=8$ \\
\hline $8^{3} \times 32$ & 64 & $0.680(94)$ & $0.113(32)$ & $0.353(63)$ & $3.38(39)$ & $1.297(63)$ & $L=8$ \\
\hline
\end{tabular}

TABLE VI: Gluonic observables obtained from the static potential for $\beta=2.3$. We note that there is no sign of volume dependence in the string tension results expressed in physical units $\sigma r_{0}^{2}$. Errors estimates are obtained from a jackknife analysis of fits.

\begin{tabular}{|c|c|c|c|c|c|c|c|}
\hline$V \times T$ & $L_{s}$ & $V_{0} a$ & $\sigma a^{2}$ & $\alpha$ & $r_{0} / a$ & $\sigma r_{0}^{2}$ & method \\
\hline $8^{3} \times 32$ & 16 & $0.617(11)$ & $0.0292(30)$ & $0.2857(83)$ & $6.84(33)$ & $1.3643(83)$ & $L=8$ \\
$16^{3} \times 32$ & 16 & $0.5846(32)$ & $0.04531(91)$ & $0.2659(24)$ & $5.526(51)$ & $1.3841(24)$ & $L=16$ \\
$16^{3} \times 32$ & 16 & $0.537(11)$ & $0.0554(20)$ & $0.219(15)$ & $5.083(67)$ & $1.431(15)$ & $L=16, r \geq \sqrt{3}$ \\
\hline $8^{3} \times 32$ & 24 & $0.636(12)$ & $0.0280(33)$ & $0.2997(88)$ & $6.94(39)$ & $1.3503(88)$ & $L=8$ \\
$16^{3} \times 32$ & 24 & $0.579(40)$ & $0.065(13)$ & $0.272(27)$ & $4.60(41)$ & $1.378(27)$ & $L=16$ \\
\hline $8^{3} \times 32$ & 32 & $0.609(12)$ & $0.0369(36)$ & $0.2809(90)$ & $6.09(28)$ & $1.369(90)$ & $L=8$ \\
$16^{3} \times 32$ & 32 & $0.611(43)$ & $0.059(13)$ & $0.295(29)$ & $4.79(50)$ & $1.355(29)$ & $L=16$ \\
\hline $8^{3} \times 32$ & 48 & $0.648(15)$ & $0.0288(44)$ & $0.309(11)$ & $6.83(49)$ & $1.341(11)$ & $L=8$ \\
\hline
\end{tabular}

TABLE VII: Gluonic observables obtained from the static potential for $\beta=2.4$. We note that $r_{0} / a$ shows significant volume dependence for $L_{s}=32$. 


\begin{tabular}{|c|c|c|}
\hline$\beta$ & $L_{s}>24$ & $L_{s}>16$ \\
\hline 2.3 & $0.00086(17)$ & $0.00026(25)$ \\
2.4 & - & $0.00098(13)$ \\
\hline
\end{tabular}

TABLE VIII: Fit results for the chiral extrapolation of the gaugino condensate, depending upon the range of $L_{s}$ values used. For $\beta=2.3$, the quality of the $L_{s}>16$ fit is very poor due to nonlinear dependence on $m_{\text {res }}$ that enters at $L_{s}=24$, as can also be seen from Fig. 3 ,

\section{EXTRAPOLATION OF THE GAUGINO CONDENSATE}

One important question is the size of $L_{s}$ necessary to get into the linear regime where

$$
\langle\bar{\lambda} \lambda\rangle \approx c_{0}+c_{1} m_{\mathrm{res}}
$$

is a good approximation. Obviously, this serves as an indicator of where we need to be in order to have SYM well-approximated. Thus, the measurement of $\langle\bar{\lambda} \lambda\rangle$ vs. $m_{\text {res }}$ is an important benchmark for determining the regime in which other SYM phenomena can be studied with the DWF lattice approach. Another question is the extent to which $c_{0,1}$ are sensitive to finite spacetime volume $\left(V_{4}=V \times T\right.$ in our notation). In fact, we find, interestingly, that most of the volume dependence is absorbed into $m_{\text {res }}$. All of this is clearly seen from Figs. 3 and 4 . One sees that to a good approximation, the $8^{3} \times 32$ and $16^{3} \times 32$ lattice data lie on the same line. The smaller value of $m_{\text {res }}$ on the smaller lattice is most likely due to a smaller density of near-zero modes. The chiral extrapolation $\left(m_{\mathrm{res}} \rightarrow 0\right)$ of $\langle\bar{\lambda} \lambda\rangle a^{3}$ obtained from the fit is given in Table VIII. A feel for the sensitivity to the fitted range of $L_{s}$ can be seen from the two results we provide for $\beta=2.3$, which differ by the minimum $L_{s}$ that was included. In fact, the quality of the $L_{s}>16$ fit is very poor due to nonlinear dependence on $m_{\text {res }}$ that enters at $L_{s}=24$, as can also be seen from Fig. 3 .

According to Table VII, the value of the lattice spacing $a$ is smaller on the $\beta=2.4$ lattice. Thus it is surprising that the extrapolated value of $\langle\bar{\lambda} \lambda\rangle a^{3}$ is larger for $\beta=2.4$ than for $\beta=2.3$. On the other hand, we note from Table III that the $\beta=2.4$ condensates measured in physical units $\left(\langle\bar{\lambda} \lambda\rangle r_{0}^{3}\right)$ are significantly larger than the $\beta=2.3$ ones, given in Table II. A plausible interpretion is that there are larger renormalizations of the condensate on a finer lattice $(\beta=2.4)$, a hypothesis that we are preparing to test with nonperturbative renormalization [42, 43] in an upcoming study. Under this interpretation we have an under- 


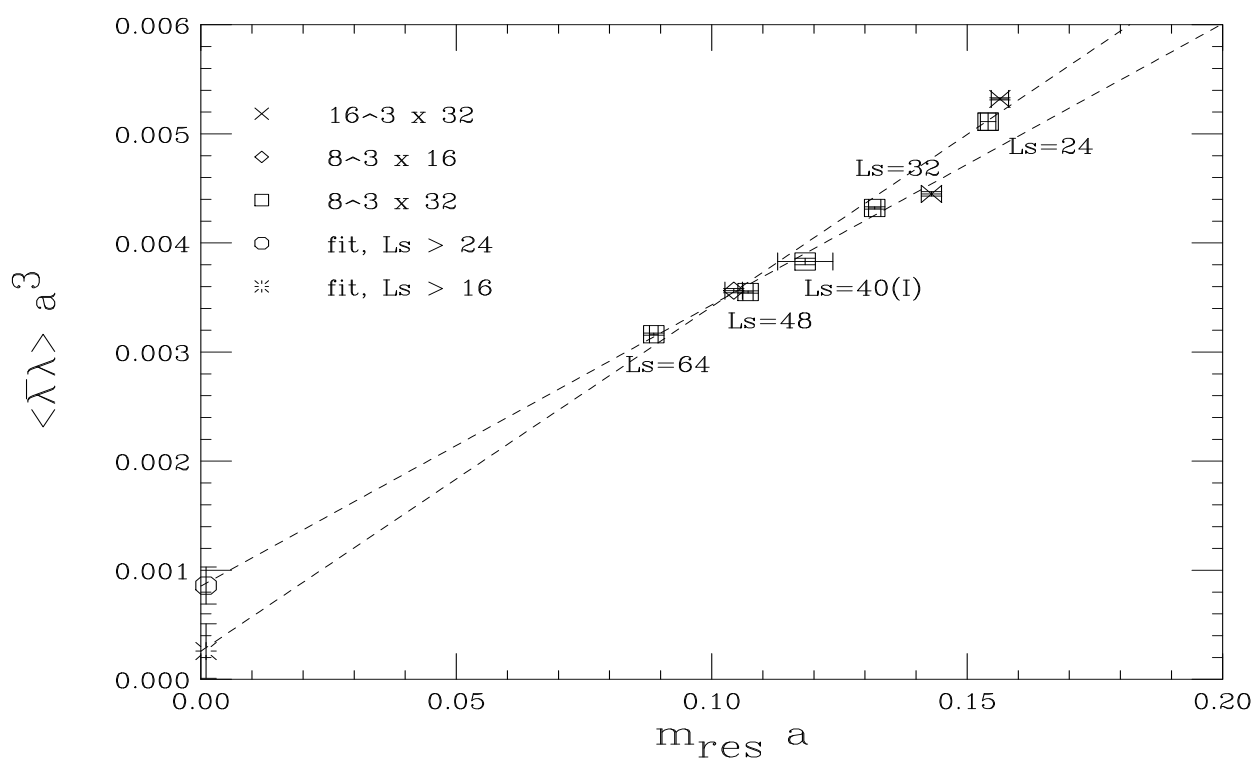

FIG. 3: Condensate vs. $m_{\text {res }}$ for $\beta=2.3$, in bare lattice units. Dashed lines show the two linear fits (differing by the minimum $L_{s}$ included). Extrapolated values together with fit errors are shown at $m_{\text {res }}=0$.

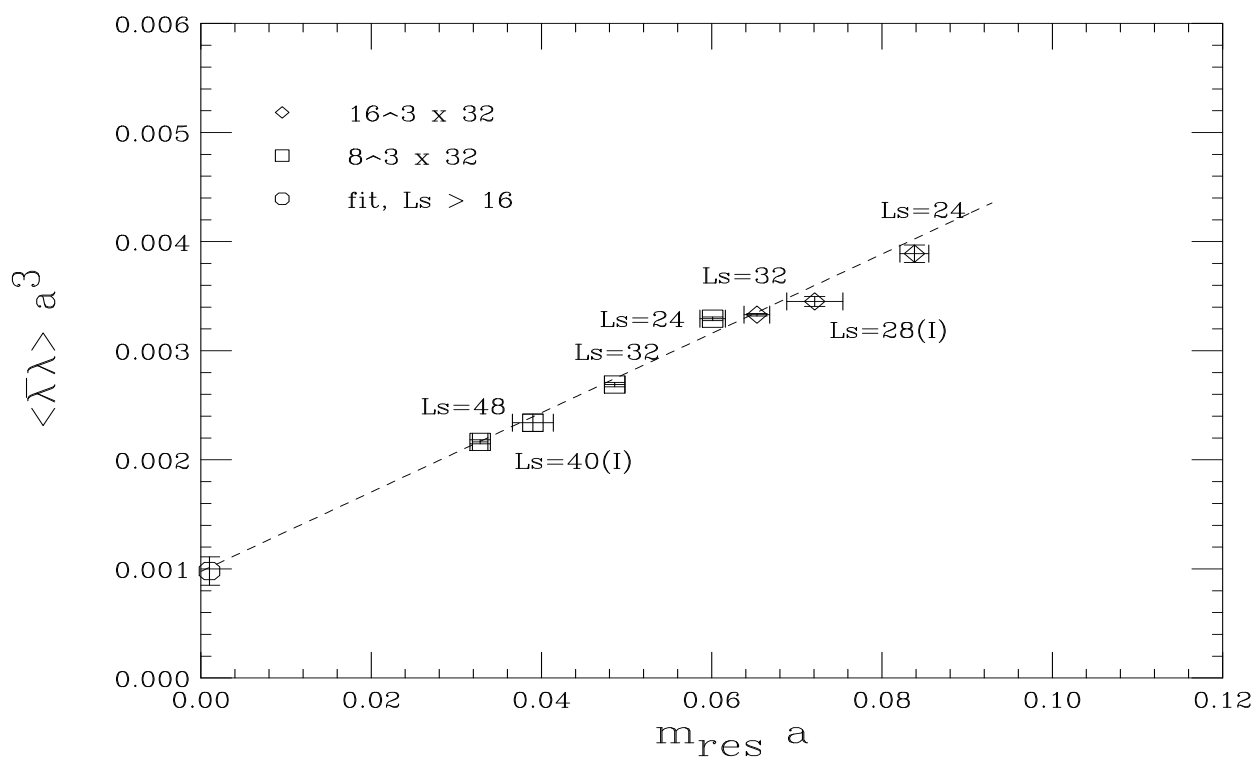

FIG. 4: Condensate vs. $m_{\text {res }}$ for $\beta=2.4$, in bare lattice units. The dashed line shows the linear fit. The extrapolated value together with fit error is shown at $m_{\text {res }}=0$. 
standing of why in Table VIII the chirally extrapolated value of the lattice units condensate for $\beta=2.4$ is larger than the one for $\beta=2.3$, which does not agree with naive expectations. It can also be seen, for instance from the $\beta=2.4, L_{s}=32$ data in Table III, that a very significant finite size effect occurs for the condensate expressed in physical units, a reflection of the finite size dependence of the static potential fit results for this choice of parameters (cf. Table VII). Finally, it is amusing that the extrapolated value of the $\beta=2.3, L_{s}>24$ fit is within $1 \sigma$ of the extrapolated value of the $\beta=2.4$ fit, though there is no reason why this should be true.

\section{CONCLUSIONS}

We have performed a detailed Monte Carlo simulation study of the supersymmetric limit of $\mathcal{N}=1, \mathrm{SU}(2)$ super-Yang-Mills using a DWF lattice formulation. Our work follows from an earlier calculation by FKV but significantly extends that work; we use larger lattices with two lattice spacings and are able to probe much closer to the chiral limit. Our results for the gaugino condensate show the correct theoretical dependence on the residual mass and allow for a reliable extrapolation to the chiral limit. Our results provide strong evidence for a nonzero gaugino condensate in the supersymmetric continuum limit, and establish important benchmarks for future studies.

Future work that is envisioned is aimed at developing a deeper understanding of the configurations that are responsible for generating the nonzero gaugino condensate. In particular, we would like to elucidate the continuum picture on the cylinder $\mathbf{R}^{3} \times S^{1}$, where it is monopoles and "KK monopoles" that combine to yield the infinite volume value [12].

At the same time, two important studies need to be done in order to further develop the lattice results presented here. First, we will make a more accurate determination of the Sommer scale $r_{0}$, using the force technique. In that study, smearing of the gauge links and other refinements appearing in [38] will be used. Second, nonperturbative renormalization of the gaugino condensate will be performed [42, 43].

Finally, we note that rather large $L_{s}$ values were required in order to get $m_{\text {res }} r_{0} \sim 1 / 4$. To improve the situation we envision switching to simulations with modified versions of DWF that have superior chiral behavior [44, 45]. 


\section{Acknowledgements}

The research described here was made possible by the supercomputing resource that was provided by Rensselaer: ten months of steady access to between one and four IBM BlueGene/L racks at the Computational Center for Nanotechnology Innovation (CCNI), for a total of approximately 30 Million IBM BlueGene/L core hours. This center was built in a three-way partnership between Rensselaer, IBM and the State of New York. Our project benefitted from reference to a copy of the 1999-2000 modified Columbia Physics System (CPS) code used by Fleming, Kogut and Vranas [34], and was built by modification of the publically available 2008 CPS lattice QCD code, developed in part under a Department of Energy SciDAC grant. J.G. thanks Adam Todorski at CCNI, and Chulwoo Jung at Columbia University and Brookhaven National Laboratory for practical help on many occasions. He

also received assistance from faculty development funds at Rensselaer. RCB's research is supported in part by the Department of Energy under contract No. DE-FG02-91ER40676. SMC is supported in part by the Department of Energy under contract No. DE-FG0285ER40237. Finally, we thank Michael Endres for correcting an error in the first version of this article.

\section{APPENDIX A: LATTICE FORMULATION}

In this appendix, the $\mathcal{N}=1, \mathrm{SU}(2) \mathrm{SYM}$ lattice action and operators associated with the gaugino condensate are described. The DWF formulation for this theory is identical to [23, 24, 34], and is described here for completeness. The lattice consists of an SU(2) gauge theory with a single Majorana fermion in the adjoint representation. As such, the fermionic part of the path integral is the analytic square root of the Dirac determinant, yielding the Pfaffian of the corresponding matrix.

The partition function is

$$
Z=\int[d U] \int[d \Psi] \int[d \Phi] e^{-S}
$$

Here $U_{\mu}(x), \mu=1,2,3,4$ is the four-dimensional gauge link field in the fundamental representation, $\Psi(x, s)$ is a (real) five-dimensional Majorana spinor in the adjoint representation and $\Phi(x, s)$ is a (real) five-dimensional bosonic Pauli Villars (PV) field with the same indices as the Majorana fermion. The coordinate $x$ denotes sites in the four-dimensional spacetime 
box, which has $L$ sites in spatial directions and $T$ sites in time. The boundary conditions along these directions are taken to be periodic for the gauge link fields but antiperiodic in time for the fermion and Pauli-Villars fields. The coordinate of the fifth direction is $s=0,1, \ldots, L_{s}-1$, where $L_{s}$ is the size of that direction and is taken to be an even number. The action $S$ is given by:

$$
S=S_{G}(U)+S_{F}(\Psi, U)+S_{P V}(\Phi, U) .
$$

$S_{G}(U)$ is the pure gauge part and is defined using the standard single plaquette action of Wilson:

$$
S_{G}=\beta \sum_{p}\left(1-\frac{1}{2} \operatorname{Re} \operatorname{Tr}\left[U_{p}\right]\right)
$$

where $\beta=4 / g^{2}$ and $g$ is the gauge coupling.

The fermion part $S_{F}(\Psi, U)$ is given by:

$$
S_{F}=-\sum_{x, x^{\prime}, s, s^{\prime}} \bar{\Psi}(x, s) D_{F}\left(x, s ; x^{\prime}, s^{\prime}\right) \Psi\left(x^{\prime}, s^{\prime}\right)
$$

where $D_{F}$ is the DWF Dirac operator in the form of [46]:

$$
\begin{gathered}
D_{F}\left(x, s ; x^{\prime}, s^{\prime}\right)=\delta\left(s-s^{\prime}\right) \not D\left(x, x^{\prime}\right)+\not D^{\perp}\left(s, s^{\prime}\right) \delta\left(x-x^{\prime}\right) \\
\not D\left(x, x^{\prime}\right)=\frac{1}{2} \sum_{\mu=1}^{4}\left[\left(1+\gamma_{\mu}\right) V_{\mu}(x) \delta\left(x+\hat{\mu}-x^{\prime}\right)+\left(1-\gamma_{\mu}\right) V_{\mu}^{\dagger}\left(x^{\prime}\right) \delta\left(x^{\prime}+\hat{\mu}-x\right)\right] \\
+\left(m_{0}-4\right) \delta\left(x-x^{\prime}\right) \\
\not D^{\perp}\left(s, s^{\prime}\right)= \begin{cases}P_{R} \delta\left(1-s^{\prime}\right)-m_{f} P_{L} \delta\left(L_{s}-1-s^{\prime}\right)-\delta\left(0-s^{\prime}\right) & s=0 \\
P_{R} \delta\left(s+1-s^{\prime}\right)+P_{L} \delta\left(s-1-s^{\prime}\right)-\delta\left(s-s^{\prime}\right) & 0<s<L_{s}-1 \\
-m_{f} P_{R} \delta\left(0-s^{\prime}\right)+P_{L} \delta\left(L_{s}-2-s^{\prime}\right)-\delta\left(L_{s}-1-s^{\prime}\right) & s=L_{s}-1\end{cases} \\
P_{R, L}=\frac{1 \pm \gamma_{5}}{2}
\end{gathered}
$$

where $V$ is the gauge field in the adjoint representation. It is related to the field in the fundamental representation:

$$
\left[V_{\mu}(x)\right]_{a, b}=\frac{1}{2} \operatorname{Tr}\left[U_{\mu}^{\dagger}(x) \sigma^{a} U_{\mu}(x) \sigma^{b}\right] .
$$

with $\sigma^{a}$ the Pauli matrices. In the above equations $m_{0}$ is a five-dimensional mass representing the "height" of the domain wall and it controls the number of light flavors in the theory. In 
order to get one light species in the free theory one must set $0<m_{0}<2$ [21]. The parameter $m_{f}$ explicitly mixes the two chiralities and as a result it controls the bare fermion mass of the four-dimensional effective theory. In our simulations we have set $m_{f}=0$.

The fermion field $\bar{\Psi}$ is not independent but is related to $\Psi$ by the equivalent of the Majorana condition for this 5-dimensional theory [24]:

$$
\bar{\Psi}=\Psi^{T} C R_{5}
$$

where $R_{5}$ is a reflection operator along the fifth direction and $C$ the charge conjugation operator in Eucledean space which can be set to:

$$
C=\gamma_{0} \gamma_{2}
$$

Therefore, the fermion action can also be written as:

$$
S_{F}=-\sum_{x, x^{\prime}, s, s^{\prime}} \Psi^{T}(x, s) M_{F}\left(x, s ; x^{\prime}, s^{\prime}\right) \Psi\left(x^{\prime}, s^{\prime}\right)
$$

where

$$
M_{F}\left(x, s ; x^{\prime}, s^{\prime}\right)=C R_{5} D_{F}\left(x, s ; x^{\prime}, s^{\prime}\right)
$$

is an antisymmetric matrix as can be easily checked [23]. As a result the fermionic integral gives the anticipated Pfaffian:

$$
\int[d \Psi] e^{-S_{F}}=\operatorname{Pf}\left(M_{F}\right)
$$

Because $\operatorname{det}\left(C R_{5}\right)=1$ one also has that $\operatorname{det}\left(M_{F}\right)=\operatorname{det}\left(D_{F}\right)$ and therefore:

$$
\operatorname{Pf}\left(M_{F}\right)=\sqrt{\operatorname{det}\left(D_{F}\right)}
$$

The Pauli-Villars (PV) action $S_{P V}$ is designed to cancel the contribution of the heavy fermions [47]. Viewing the extra dimension as an internal flavor space [47] one can see that there are $L_{s}-1$ heavy fermions with masses near the cutoff and one light fermion. The PV subtraction removes effects of the $L_{s}$ heavy particles in such a way that the overlap determinant is obtained in the $L_{s} \rightarrow \infty$ limit [23]. The PV subtraction used here is as in [48] and is given by:

$$
S_{P V}=\sum_{x, x^{\prime}, s, s^{\prime}} \Phi^{T}(x, s) M_{F}\left[m_{f}=1\right]\left(x, s ; x^{\prime}, s^{\prime}\right) \Phi\left(x^{\prime}, s^{\prime}\right) .
$$


The integral over the PV fields results in:

$$
\int[d \Phi] e^{-S_{P V}}=\frac{1}{\operatorname{Pf}\left(M_{F}\left[m_{f}=1\right]\right)} .
$$

The gaugino condensate is measured using four-dimensional fermion fields that are a projection of the five-dimensional DWF [46]:

$$
\begin{aligned}
& \chi(x)=P_{R} \Psi(x, 0)+P_{L} \Psi\left(x, L_{s}-1\right) \\
& \bar{\chi}(x)=\bar{\Psi}\left(x, L_{s}-1\right) P_{R}+\bar{\Psi}(x, 0) P_{L} .
\end{aligned}
$$

In the $L_{s} \rightarrow \infty$ limit of the theory these operators directly correspond to insertions in the overlap of appropriate creation and annihilation operators [47].

Using Eq. (A10) and (A18) the Majorana condition on the four-dimensional fermion field is:

$$
\bar{\chi}=\chi^{T} C
$$

Because this is the correct condition for a four-dimensional field one can see that the definition in Eq. (A10) not only produces an antisymmetric fermion matrix $M_{F}$ but is also consistent with the projection prescription in Eq. (A18) as expected.

\section{APPENDIX B: RESIDUAL MASS}

Residual chiral symmetry breaking is understood through the axial Ward identity on the DWF lattice [36], which we write here for the case of vanishing bare mass:

$$
\nabla_{\mu}\left\langle A_{\mu}^{a}(x) P^{b}(0)\right\rangle=2\left\langle J_{5 q}^{a}(x) P^{b}(0)\right\rangle
$$

where $A_{\mu}^{a}$ is a DWF version of the axial current with isospin index $a=1,2,3$ and $P^{a}$ is a DWF version of the corresponding pseudoscalar current, the interpolating operator for the "pion." We note that in the present case the pion is in the adjoint representation of the $\mathrm{SU}(2)$ gauge group (not to be confused with isospin). The pseudoscalar current $J_{5 q}^{a}$ is different in that it represents a pion at the middle of the fifth dimension:

$$
J_{5 q}^{a}(x)=-\bar{\psi}\left(x, L_{s} / 2-1\right) P_{L} \sigma^{a} \psi\left(x, L_{s} / 2\right)+\bar{\psi}\left(x, L_{s} / 2\right) P_{R} \sigma^{a} \psi\left(x, L_{s} / 2-1\right) .
$$

It accounts for the difference in how the left- and right-handed "quark" fields in the DWF description transform, which causes a mismatch midway between the domain walls and hence the explicit nonconservation of the axial current in the lattice theory at finite $L_{s}$. 
Since in the continuum limit the Ward identity must transition to the continuum form, we see that $J_{5 q}$ must be related to the pseudoscalar current $P$ through the residual mass that is a consequence of $L_{s} \neq \infty$ :

$$
J_{5 q}^{a} \approx m_{\mathrm{res}} P^{a}
$$

Thus to extract the residual chiral symmetry breaking one studies the large (imaginary) time behavior of the ratio:

$$
m_{\mathrm{res}}=\lim _{t \rightarrow \infty} \frac{\sum_{\vec{x}, \vec{y}} J_{5 q}^{a}(t, \vec{x}) P^{a}(0, \vec{y})}{\sum_{\vec{x}, \vec{y}} P^{a}(t, \vec{x}) P^{a}(0, \vec{y})} .
$$

In all of our work we find that this quantity reaches a plateau in the range $3<t<T-2$, and fit to a constant in that region of $t$.

\section{APPENDIX C: SIMULATION}

Here we make a few brief remarks on the computational aspects of this project. Configurations were generated with the rational hybrid Monte Carlo algorithm [49, 50, 51]. All simulations were performed on Rensselaer's Computational Center for Nanotechnology Innovations cluster of 16 IBM BlueGene/L machines. We typically used the full capacity of two such machines 24 hours/day, and generated configurations for approximately ten months, for a total of approximately 30 Million IBM BlueGene/L core hours. The time required for data analysis was a small fraction of this, by comparison. Naturally, the large lattices $\left(16^{3} \times 32\right)$ with small $m_{\text {res }}$ values were the most costly to generate.

In the rational approximation used to generate configurations, we found that it was necessary to go to rather high degrees, due to a very wide spread between lowest and highest eigenvalues of the Dirac operator. This occured because we performed our simulations at vanishing bare fermion mass $m_{f}=0$, relying on the finite but large value of $L_{s}$ as an infrared regulator. Typically, the Metropolis step required degrees between 15 and 20 in the computation of the change in the Hamiltonian, in order to have sufficient accuracy. Moreover, it was not unusual to require between 50 and 100 steps in the leapfrog integration for a trajectory of $\tau=0.5$ simulation time units, in order to get reasonable acceptance rates at large $L_{s}$. Again, this was a result of small eigenvalues of the Dirac operator. Naturally, these features led to very slow updating. For the $L_{s}$ values that we simulated, a 


\begin{tabular}{|c|c|c|c|c|}
\hline$\beta$ & $V \times T$ & $L_{s}$ & $N_{\tau}$ & configs./day \\
\hline 2.3 & $8^{3} \times 32$ & 48 & 60 & 140 \\
2.3 & $8^{3} \times 32$ & 64 & 80 & 70 \\
2.3 & $16^{3} \times 32$ & 32 & 90 & 40 \\
2.4 & $8^{3} \times 32$ & 48 & 100 & 80 \\
2.4 & $16^{3} \times 32$ & 48 & 200 & 11 \\
\hline
\end{tabular}

TABLE IX: Example timing results on a single BlueGene/L rack (1024 dual core nodes). Here, $N_{\tau}$ is the number of steps in the leapfrog trajectory.

single BlueGene/L rack was able to produce $\mathcal{O}(10)$ to $\mathcal{O}(100)$ configurations per day. Some examples are given in Table IX. The last row represents a run that was not reported in the main text as it was too slow for a reasonable data set to be generated in a practical time-frame. As a result, our forthcoming work will set $m_{f} \neq 0$ and perform an $m_{f} \rightarrow 0$ extrapolation for these larger volume, large $L_{s}$ simulations.

[1] J. Giedt, "Deconstruction and other approaches to supersymmetric lattice field theories," Int. J. Mod. Phys. A 21 (2006) 3039 arXiv:hep-lat/0602007.

[2] S. Catterall, "Dirac-Kähler fermions and exact lattice supersymmetry," PoS LAT2005 (2006) 006 arXiv:hep-lat/0509136.

[3] P. H. Dondi and H. Nicolai, "Lattice Supersymmetry," Nuovo Cim. A 41 (1977) 1.

[4] S. Ferrara and B. Zumino, "Supergauge Invariant Yang-Mills Theories," Nucl. Phys. B 79 (1974) 413.

[5] G. Curci and G. Veneziano, "Supersymmetry And The Lattice: A Reconciliation?," Nucl. Phys. B 292 (1987) 555.

[6] P. H. Ginsparg and K. G. Wilson, "A Remnant Of Chiral Symmetry On The Lattice," Phys. Rev. D 25 (1982) 2649.

[7] M. Luscher, "Exact chiral symmetry on the lattice and the Ginsparg-Wilson relation," Phys. Lett. B 428 (1998) 342 arXiv:hep-lat/9802011.

[8] G. Veneziano and S. Yankielowicz, "An Effective Lagrangian For The Pure N=1 Supersym- 
metric Yang-Mills Theory,” Phys. Lett. B 113 (1982) 231.

[9] I. Affleck, M. Dine and N. Seiberg, "Dynamical Supersymmetry Breaking In Supersymmetric QCD," Nucl. Phys. B 241 (1984) 493.

[10] I. Affleck, M. Dine and N. Seiberg, "Dynamical Supersymmetry Breaking In Four-Dimensions And Its Phenomenological Implications," Nucl. Phys. B 256 (1985) 557.

[11] V. A. Novikov, M. A. Shifman, A. I. Vainshtein and V. I. Zakharov, "Supersymmetric instanton calculus: Gauge theories with matter,” Nucl. Phys. B 260 (1985) 157 [Yad. Fiz. 42 (1985) 1499].

[12] N. M. Davies, T. J. Hollowood, V. V. Khoze and M. P. Mattis, "Gluino condensate and magnetic monopoles in supersymmetric gluodynamics," Nucl. Phys. B 559 (1999) 123 arXiv:hep-th/9905015.

[13] F. Cachazo, M. R. Douglas, N. Seiberg and E. Witten, "Chiral rings and anomalies in supersymmetric gauge theory," JHEP 0212 (2002) 071 arXiv:hep-th/0211170.

[14] N. J. Evans, S. D. H. Hsu and M. Schwetz, "Lattice tests of supersymmetric Yang-Mills theory?," arXiv:hep-th/9707260.

[15] G. R. Farrar, G. Gabadadze and M. Schwetz, "On the effective action of N = 1 supersymmetric Yang-Mills theory," Phys. Rev. D 58 (1998) 015009 arXiv:hep-th/9711166.

[16] G. R. Farrar, G. Gabadadze and M. Schwetz, "The spectrum of softly broken N = 1 supersymmetric Yang-Mills theory," Phys. Rev. D 60 (1999) 035002 arXiv:hep-th/9806204.

[17] G. Gabadadze, "The discrete $\mathrm{Z}(2 \mathrm{~N}(\mathrm{c}))$ symmetry and effective superpotential in SUSY gluodynamics," Nucl. Phys. B 544 (1999) 650 arXiv:hep-th/9808005.

[18] D. G. Cerdeno, A. Knauf and J. Louis, "A note on effective N = 1 super Yang-Mills theories versus lattice results," Eur. Phys. J. C 31 (2003) 415 arXiv:hep-th/0307198.

[19] P. Merlatti and F. Sannino, "Extending the Veneziano-Yankielowicz effective theory," Phys. Rev. D 70 (2004) 065022 arXiv:hep-th/0404251.

[20] R. Auzzi and F. Sannino, "Hidden modulus in the extended Veneziano-Yankielowicz theory," Phys. Rev. D 72 (2005) 105003 arXiv:hep-th/0509166].

[21] D. B. Kaplan, "A Method for simulating chiral fermions on the lattice," Phys. Lett. B 288 (1992) 342 arXiv:hep-lat/9206013.

[22] Y. Shamir, "Chiral fermions from lattice boundaries," Nucl. Phys. B 406 (1993) 90 arXiv:hep-lat/9303005. 
[23] H. Neuberger, "Vector like gauge theories with almost massless fermions on the lattice," Phys. Rev. D 57 (1998) 5417 arXiv:hep-lat/9710089].

[24] D. B. Kaplan and M. Schmaltz, "Supersymmetric Yang-Mills theories from domain wall fermions," Chin. J. Phys. 38 (2000) 543 arXiv:hep-lat/0002030].

[25] I. Campos, R. Kirchner, I. Montvay, J. Westphalen, A. Feo, S. Luckmann, G. Münster, K. Spanderan [DESY-Munster Collaboration], "Monte Carlo simulation of SU(2) Yang-Mills theory with light gluinos," Eur. Phys. J. C 11 (1999) 507 arXiv:hep-lat/9903014].

[26] F. Farchioni, C. Gebert, R. Kirchner, I. Montvay, A. Feo, G. Münster, T. Galla, A. Vladikas [DESY-Munster-Roma Collaboration], "The supersymmetric Ward identities on the lattice," Eur. Phys. J. C 23 (2002) 719 arXiv:hep-lat/0111008]; I. Montvay, "Supersymmetric YangMills theory on the lattice," Int. J. Mod. Phys. A 17 (2002) 2377 arXiv:hep-lat/0112007]; R. Peetz, F. Farchioni, C. Gebert and G. Munster, "Spectrum of SU(2) SUSY Yang-Mills theory with a light gluino," Nucl. Phys. Proc. Suppl. 119 (2003) 912 arXiv:hep-lat/0209065].

[27] F. Farchioni, G. Muenster and R. Peetz, "The volume source technique for flavor singlets: A second look," Eur. Phys. J. C 38 (2004) 329 arXiv:hep-lat/0404004.

[28] F. Farchioni and R. Peetz, "The low-lying mass spectrum of the N=1 SU(2) SUSY Yang-Mills theory with Wilson fermions," Eur. Phys. J. C 39 (2005) 87 arXiv:hep-lat/0407036.

[29] R. Peetz, "Spectrum of $\mathcal{N}=1$ Super Yang Mills Theory on the Lattice with a Light Gluino," doctoral dissertation, University of Münster, Germany, 2003, available at: http://deposit.ddb.de/cgi-bin/dokserv?idn=97018249X.

[30] I. Montvay, Nucl. Phys. B83-84 (Proc. Suppl) (2000) 188; DESY-Münster Collaboration (A. Feo et al.), Nucl. Phys. B83-84 (Proc. Suppl) (2000) 661; DESY-Münster Collaboration (A. Feo et al.), Nucl. Phys. B83-84 (Proc. Suppl) (2000) 670; DESY-Münster Collaboration (R. Kirchner et al.), Phys. Lett. B446 (1999) 209; G. Koutsoumbas et. al. B63 (Proc. Suppl) (1998) 727; I. Montvay B63 (Proc. Suppl) (1998) 108; G. Koutsoumbas, I. Montvay, Phys. Lett. B398 (1997) 130; I. Montvay, Nucl. Phys. B53 (Proc. Suppl) (1997) 853; I. Montvay, Nucl. Phys. B466 (1996) 259.

[31] A. Donini, M. Guagnelli, P. Hernandez and A. Vladikas, "Towards N = 1 Super-YangMills on the lattice," Nucl. Phys. B 523 (1998) 529 arXiv:hep-lat/9710065; A. Donini, E. Gabrielli and M. B. Gavela, "Quenched supersymmetry," Nucl. Phys. B 546 (1999) 119 arXiv:hep-th/9810127]; A. Donini and M. Guagnelli, "Hybrid molecular dynamics for lattice 
supersymmetry," Phys. Lett. B 383 (1996) 301 arXiv:hep-lat/9605010.

[32] Kamel Demmouche et al., "Spectrum of 4d N=1 SYM on the lattice with light dynamical gluinos," in proceedings "The XXVI International Symposium of Lattice Field Theory," College of William and Mary, Williamsburg, VA, July 14-19, 2008, PoS (LATTICE 2008) 061.

[33] J. Giedt, R. Brower, S. Catterall, G. T. Fleming, P. Vranas, "Gluinos condensing at the CCNI: 4096 CPUs weigh in," in proceedings "Continuous Advances in QCD 2008," Fine Theoretical Physics Institute, University of Minnesota, Minneapolis, MN, May 15-18, 2008, ed. M. Peloso, World Scientific, Singapore arXiv:0807.2032]; "Domain wall fermion lattice super-Yang-Mills," in proceedings "The XXVI International Symposium of Lattice Field Theory," College of William and Mary, Williamsburg, VA, July 14-19, 2008, PoS (LATTICE 2008) 053.

[34] G. T. Fleming, J. B. Kogut and P. M. Vranas, "Super Yang-Mills on the lattice with domain wall fermions," Phys. Rev. D 64 (2001) 034510 arXiv:hep-lat/0008009.

[35] M. Endres, "Numerical simulation of $\mathcal{N}=1$ supersymmetric Yang-Mills theory," in proceedings "The XXVI International Symposium of Lattice Field Theory," College of William and Mary, Williamsburg, VA, July 14-19, 2008, PoS (LATTICE 2008) 025 [arXiv:0810.0431].

[36] T. Blum et al., "Quenched lattice QCD with domain wall fermions and the chiral limit," Phys. Rev. D 69 (2004) 074502 arXiv:hep-lat/0007038.

[37] Y. Shamir, "New domain-wall fermion actions," Phys. Rev. D 62 (2000) 054513 arXiv:hep-lat/0003024.

[38] R. Sommer, "A New way to set the energy scale in lattice gauge theories and its applications to the static force and alpha-s in SU(2) Yang-Mills theory," Nucl. Phys. B 411 (1994) 839 arXiv:hep-lat/9310022.

[39] J. Giedt, "Residual mass measurements in domain wall fermion lattice super-Yang-Mills," in progress.

[40] F. Farchioni, I. Montvay, G. Munster, E. E. Scholz, T. Sudmann and J. Wuilloud, "Hadron spectrum of QCD with one quark flavor," arXiv:0810.0161 [hep-lat].

[41] M. Creutz, "Asymptotic Freedom Scales," Phys. Rev. Lett. 45 (1980) 313.

[42] G. Martinelli, C. Pittori, C. T. Sachrajda, M. Testa and A. Vladikas, "A General Method For Nonperturbative Renormalization Of Lattice Operators," Nucl. Phys. B 445 (1995) 81 arXiv:hep-lat/9411010. 
[43] T. Blum et al., "Non-perturbative renormalisation of domain wall fermions: Quark bilinears," Phys. Rev. D 66 (2002) 014504 arXiv:hep-lat/0102005].

[44] P. M. Vranas, "Gap domain wall fermions," Phys. Rev. D $\mathbf{7 4}$ (2006) 034512 arXiv:hep-lat/0606014].

[45] R. C. Brower, H. Neff and K. Orginos, "Moebius fermions: Improved domain wall chiral fermions," Nucl. Phys. Proc. Suppl. 140 (2005) 686 [arXiv:hep-lat/0409118].

[46] V. Furman and Y. Shamir, "Axial Symmetries In Lattice QCD With Kaplan Fermions," Nucl. Phys. B 439 (1995) 54 arXiv:hep-lat/9405004.

[47] R. Narayanan, H. Neuberger, Phys. Lett. B302 (1993) 62; Phys. Rev. Lett. 71 (1993) 3251; Nucl. Phys. B412 (1994) 574; Nucl. Phys. B443 (1995) 305.

[48] P.M. Vranas, Lattice 96, Nucl. Phys. B53 (Proc. Suppl.) (1997) 278; Phys. Rev. D57 (1998) 1415 .

[49] I. Horváth, A. D. Kennedy and S. Sint, "A new exact method for dynamical fermion computations with nonlocal action," Nucl. Phys. (Proc. Suppl.) B73 (1999) 834.

[50] M. A. Clark and A. D. Kennedy, "The RHMC algorithm for two flavors of dynamical staggered fermions," Nucl. Phys. (Proc. Suppl.) B129 (2004) 850.

[51] M. A. Clark, A. D. Kennedy and Sroczynski, "Exact 2+1 flavor RHMC simulations,' Nucl. Phys. (Proc. Suppl.) B140 (2005) 835. 